Review Article

\title{
Mechanobiology of Periodontal Ligament Stem Cells in Orthodontic Tooth Movement
}

\author{
Huaming Huang $\mathbb{D}$, Ruili Yang $\mathbb{D}$, and Yan-heng Zhou \\ Department of Orthodontics, Peking University School and Hospital of Stomatology, National Engineering Laboratory for Digital and \\ Material Technology of Stomatology, Beijing Key Laboratory of Digital Stomatology, Beijing 100081, China
}

Correspondence should be addressed to Ruili Yang; ruiliyangabc@163.com and Yan-heng Zhou; yanhengzhou@vip.163.com

Received 12 June 2018; Revised 28 July 2018; Accepted 12 August 2018; Published 17 September 2018

Academic Editor: Philippe Bourin

Copyright (c) 2018 Huaming Huang et al. This is an open access article distributed under the Creative Commons Attribution License, which permits unrestricted use, distribution, and reproduction in any medium, provided the original work is properly cited.

\begin{abstract}
Periodontal ligament stem cells (PDLSCs) possess self-renewal, multilineage differentiation, and immunomodulatory properties. They play a crucial role in maintaining periodontal homeostasis and also participated in orthodontic tooth movement (OTM). Various studies have applied controlled mechanical stimulation to PDLSCs and investigated the effects of orthodontic force on PDLSCs. Physical stimuli can regulate the proliferation and differentiation of PDLSCs. During the past decade, a variety of studies has demonstrated that applied forces can activate different signaling pathways in PDLSCs, including MAPK, TGF- $\beta$ / Smad, and Wnt/ $\beta$-catenin pathways. Besides, recent advances have highlighted the critical role of orthodontic force in PDLSC fate through mediators, such as IL-11, CTHRC1, miR-21, and $\mathrm{H}_{2} \mathrm{~S}$. This perspective review critically discusses the PDLSC fate to physical force in vitro and orthodontic force in vivo, as well as the underlying molecular mechanism involved in OTM.
\end{abstract}

\section{Introduction}

Orthodontic tooth movement (OTM) is induced by mechanical forces and is promoted by the remodeling of periodontal ligament (PDL) and alveolar bone. Under the stimulation of appropriate orthodontic force, the periodontal tissue is reconstructed at the molecular, cellular, and tissue levels [1]. On the compression side, the PDL becomes compressed and disorganized and it results in bone resorption, while the stretching of PDL fibers induces bone deposition on the tension side [2].

Orthodontists successfully correct malocclusion by orthodontic treatment. However, there are still some challenges for them. For example, excessive orthodontic forces or common forces on the teeth of periodontitis patients may cause periodontium damage. What is more, mechanical force-induced orthodontic root resorption and relapse after treatment are still major clinical challenges in orthodontic treatment.

Since 2004, periodontal ligament stem cells (PDLSCs) have been separated and shown to share characteristics with mesenchymal stem cells (MSCs) [3]. Having immunomodulatory function and potential for proliferation and generation of cementum/periodontal ligament-like complex [3, 4], PDLSCs play important roles in periodontal homeostasis. And they are likely sensitive to mechanical loading and play critical roles in periodontal and osseous remodeling during OTM. A better understanding of the mechanical response of PDLSCs and the cellular signaling pathway involved may help in solving the challenges in orthodontic treatment.

The role of PDLSCs responding to orthodontic force during the tooth movement in vivo has been confirmed [5]. In vitro, recent advances have also clarified that the applied mechanical force, including tension, compression, and vibration, can significantly regulate the proliferation and differentiation of cultured PDLSCs. The data is summarized in Table 1 to compare expediently the response of PDLSCs to different mechanical force and summarize the effect that the duration, frequency, and magnitude have on cellular fate, which may help in understanding why low force is critical in achieving physical bone remodeling in orthodontic 
TABLE 1: Effects of applied force induced the function of PDLSCs.

\begin{tabular}{|c|c|c|c|c|}
\hline Force types & Cell source & Culture & $\begin{array}{l}\text { Mechanical devices } \\
\text { and parameter }\end{array}$ & Discoveries \\
\hline
\end{tabular}

Tension

Compression

Vibration

$\begin{array}{lcc}\text { Ultrasound [14] } & \begin{array}{c}\text { Six-week-old male } \\ \text { Wistar Han rats }\end{array} & \begin{array}{c}\text { In } \alpha \text {-MEM; on } \\ \text { 6-well plates }\end{array} \\ \text { Microgravity [15] } & \begin{array}{c}\text { Premolars and } \\ \text { third molars }\end{array} & \begin{array}{c}\text { In DMEM; on } \\ \text { Cytodex 3 microcarriers }\end{array}\end{array}$

treatment. In this review, we include the studies focusing on PDLSCs instead of periodontal ligament cells (PDLCs) isolated according to cell culture methods and identification of the multipotency of stem cells [5-15]. In order to find out the role of PDLSCs in OTM, we expound the response of PDLSCs to orthodontic force in vivo and mechanical force in vitro and also summarized the critical related mechanosensors and mechanism pathways.
Self-made four-point bending system; cyclic tension; $0.5 \mathrm{~Hz}$, $0.3 \%, 3 \mathrm{~h}, 6 \mathrm{~h}, 12 \mathrm{~h}$, and $24 \mathrm{~h}$

Flexcell FX-4000T Tension

Plus System; cyclic tension,

$0.1 \mathrm{~Hz}, 12 \%, 6 \mathrm{~h}, 12 \mathrm{~h}$, and $24 \mathrm{~h}$

Custom-built bioreactor system; cyclic tension, $0.5 \mathrm{~Hz}, 5 \%, 2 \mathrm{~h}$

Flexcell Tension System; dome-shaped equibiaxial static mechanics

Flexcell FX-4000 T Tension Plus System; static tension, $0.1 \mathrm{~Hz}, 6 \%, 8 \%, 10 \%, 12 \%$, and $14 \%, 12 \mathrm{~h}$

A layer of glass cover and metal weights; static compression, $1 \mathrm{~g} / \mathrm{cm}^{2}$, cultured for $12 \mathrm{~h}$ and $24 \mathrm{~h}$ after force withdrawal

Hydraulic pressure-controlled cellular strain unit; $1000 \mathrm{~g} / \mathrm{cm}^{2}, 1 \mathrm{~h}$ and $12 \mathrm{~h}$

GJX-5 vibration sensor; $10-180 \mathrm{~Hz}, 0.3 \mathrm{~g}, 30 \mathrm{mins} / 24 \mathrm{~h}$
GJX-5 vibration sensor; $50 \mathrm{~Hz}, 0.05 \mathrm{~g}$ to $0.9 \mathrm{~g}$, $30 \mathrm{mins} / 24 \mathrm{~h}$

A DuoSon therapeutic ultrasound device; $1 \mathrm{MHz}$, 5 or 20 mins

Rotating bioreactor; $15 \mathrm{rpm}, 24 \mathrm{~h}$
Increased osteogenic markers

Increased osteogenic markers, decreased proliferation

Increased markers of cardiomyogenesis

Increased keratocyte markers

Optimal magnitude in promoting proliferation and osteogenic activity is $12 \%$ for HPDLSCs and $8 \%$ for PPDLSCs

Altered cell morphology and repressed collagen expression, which both recovered after force withdrawal

Increased and reduced osteogenic markers after $1 \mathrm{~h}$ and 12; reduced and upregulated ratios of RANKL and OPG after $1 \mathrm{~h}$ and $12 \mathrm{~h}$, respectively

Decreased proliferation and increased osteogenic markers in a frequency-dependent manner, with significant peaks at $50 \mathrm{~Hz}$

Decreased proliferation and increased osteogenic markers in magnitude-dependent manners, with significant peaks at $0.3 \mathrm{~g}$; no obvious senescent cells

Increased proliferation

Alterations of morphology, increased proliferation, and osteogenic differentiation

\section{The Effects of Orthodontic Force on PDLSCs during the Tooth Movement In Vivo}

Orthodontic tooth movement is induced by the constant application of orthodontic force, which is promoted by PDL and alveolar bone remodeling. The force exerted on the teeth is transferred to the alveolar bone through PDL. It is generally agreed that compression causes bone resorption, 
which is regarded as the rate-limiting step, while tension leads to bone formation [1]. Several studies have explored the role of PDLSCs during OTM in vivo.

Zhang and colleagues established an OTM rat model and used PDGFR $\alpha$ and nestin to track the response of rat PDLSCs (rPDLSCs) in vivo [5]. They found that after 3 days of orthodontic treatment, the number of PDGFR $\alpha$ or nestin-positive cells increased on both of the compression and of the tension sides and then dropped after 7 days, suggesting that rPDLSCs may be reactivated on both sides during orthodontic force treatment.

Besides, rPDLSCs play a role in PDL recovery and orthodontic relapse process. After the orthodontic force is removed, PDL can return to its original structure and then orthodontic relapse occurs [16]. Feng and colleagues demonstrated that upon orthodontic force, the density of PDL collagen, as an important component of extracellular matrix in PDL, reduced on the compression side and recovered after force was removed for 5 days [6]. Correspondingly, the expression of type I collagen (Col-I) in rPDLSCs was declined with orthodontic force applied and recovered after force removal.

\section{The Molecules Transmitting the Orthodontic Force to PDLSCs In Vivo}

During OTM, a variety of molecules help in transducing the force signals into PDLSCs, such as interleukin- (IL-) 11, collagen triple helix repeat containing 1 (CTHRC1), microRNA-21 (miR-21), and hydrogen sulfide $\left(\mathrm{H}_{2} \mathrm{~S}\right)$.

IL-11 is produced by a variety of PDLCs, including fibroblasts, osteoclasts, and osteoblasts [17]. It is known to be associated in the regulation of osteoclasts and osteoblasts as well as bone remodeling [18]. After OTM, it was observed that IL-11 increased in the rat PDL [19]. Correspondingly, IL-11 could stimulate osteoblastic markers and cementoblast-specific markers, increase the expression of bone sialoprotein, and promote the proliferation of human PDLSCs (hPDLSCs). These indicated that force-induced IL-11 was secreted by PDLCs and it was good for hPDLSCs to differentiate into osteoblasts or cementoblasts [19].

CTHRC1 plays an important role in the differentiation of bone MSCs [20]. Wang and colleagues showed that the expression of CTHRC1 was upregulated in PDLCs during OTM and the osteogenic differentiation of hPDLSCs could be promoted by the overexpression of CTHRC1 in vitro, demonstrating that osteogenic differentiation of hPDLSCs was positively regulated by CTHRC1 during OTM [21].

MicroRNAs, small noncoding RNAs, could be mechanically sensitive and act as key posttranscriptional regulators in osteogenic differentiation of PDLSCs following orthodontic force. Microarray data indicated that 53 microRNAs in hPDLSCs were differentially expressed after tension [22], including hsa-miR-21, which was found to be involved in tension-induced osteogenesis of hPDLSCs in vitro [23]. Using wild and miR-21-deficient (miR-21 ${ }^{-1-}$ ) OTM model of rats, Chen and colleagues observed that miR-21 improved force-induced alveolar bone formation on the tension side during OTM in wild rats, which was suppressed in miR-
$21^{-1-}$ rats [24]. Furthermore, for the first time, hPDLSCs were collected from donors with or without OTM. The expression of miR-21 was upregulated with promoted osteogenesis in the cultured hPDLSCs following OTM, which was blocked by the inhibition of miR-21. Interestingly, even after the removal of orthodontic force, the increase in cultured hPDLSC osteogenesis was preserved, suggesting an epigenetic effect.

$\mathrm{H}_{2} \mathrm{~S}$ is a gaseous transmitter that has recently been associated with the function of MSCs and bone metabolism [25]. It has been elucidated that the production of forceinduced $\mathrm{H}_{2} \mathrm{~S}$ in hPDLSCs modulated the accumulation of macrophage and osteoclastic and osteogenic activities in the alveolar bone through regulation of the secretion of monocyte chemoattractant protein-1 and the receptor activator of the nuclear factor- $\kappa \mathrm{B}$ ligand/osteoprotegerin (RANKL/ OPG) system and then controlled the process of OTM [26]. Using a mouse OTM model, Liu and colleagues observed that orthodontic force application elevated the production of $\mathrm{H}_{2} \mathrm{~S}$ and upregulated cystathionine $\beta$-synthase (CBS) in PDL. Moreover, most of the expression of CBS was colocalized with CD90 (an MSC marker). Correspondingly, the secretion of compression-induced $\mathrm{H}_{2} \mathrm{~S}$ in the supernatant of cultured hPDLSCs was associated with the CBS expression change in hPDLSCs. Furthermore, blocking the production of endogenous $\mathrm{H}_{2} \mathrm{~S}$ suppressed the orthodontic force-induced macrophage accumulation and osteoblasts on the tension side and osteoclasts on the compression side, with a decrease in the distance of OTM. The study showed that PDLSCs generated $\mathrm{H}_{2} \mathrm{~S}$ to transduce and respond to force stimulation.

\section{The Effects of Mechanical Force on the Function of PDLSCs In Vitro}

To study the mechanobiology of PDLSCs during OTM, lots of studies applied tension and compression mimicking the force on both sides of teeth during OTM to cultured PDLSCs in vitro. And other types of mechanical stimulation also have been reported, including vibration, ultrasound, and microgravity (Table 1).

4.1. Tension. It has been reported that tension was important for the regulation of ligament tissue remodeling [27]. In one study, the tension of $3000 \mu$ strain (nearly $0.3 \%$ ) at $0.5 \mathrm{~Hz}$ was applied to hPDLSCs for $3 \mathrm{~h}, 6 \mathrm{~h}, 12 \mathrm{~h}$, and $24 \mathrm{~h}$ [7]. The expression of runt-related transcription factor-2, osterix, and Satb2 was significantly upregulated in hPDLSCs in a time-dependent manner, indicating an early response to osteogenic orientation. Other groups also confirmed that tension induced late-stage osteogenic transcription markers, such as osteocalcin [8].

PDLSCs transform tension into different cell behaviors, depending on the manner in which tension is applied, including mechanical devices, magnitude, duration, and frequency. When Pelaez and colleagues applied 5\% tension at $0.5 \mathrm{~Hz}$ for $2 \mathrm{~h}$ to hPDLSCs, they exhibited induced expression of cardiac-specific transcription factors [9]. Another study found that the dome-shaped tension promoted PDLSCs to 
differentiate into keratocytes, which had synergistic effects with induction medium [10].

To investigate the effect of tension on the function of PDLSCs to regulate osteoclastogenesis, PDLSCs were applied to static mechanical strain (SMS) with a range of magnitudes from $6 \%$ to $14 \%$ at $0.1 \mathrm{~Hz}$ for $12 \mathrm{~h} \mathrm{[11].} \mathrm{When} \mathrm{the} \mathrm{strain} \mathrm{was}$ less than $12 \%$, the osteoclastic genes (RANKL) showed no significant differences. However, when the strain was higher than $12 \%$ in PDLSCs, the levels of osteoclastic genes were obviously increased [11].

What is more, PDLSCs obtained from periodontitis patients (PPDLSCs) and healthy donors (HPDLSCs) respond differently to tension. When PPDLSCs and HPDLSCs were exposed to SMS with a range of magnitudes from $6 \%$ to $14 \%$ at $0.1 \mathrm{~Hz}$ for $12 \mathrm{~h}$, Liu and colleagues observed that different magnitudes of SMS exerted distinct effects on HPDLSCs and PPDLSCs [11]. For HPDLSCs, the best SMS value for the balance between osteogenesis and osteoclastogenesis was $12 \%$, while the optimal force for PPDLSCs was $8 \%$. Excessive SMS would damage the function of both HPDLSCs and PPDLSCs. Furthermore, compared to HPDLSCs, PPDLSCs showed decreased osteogenic activity, activated osteoclastogenesis, and greater secretion of inflammatory cytokines, which indicated that PPDLSCs are more sensitive and less tolerant to SMS.

The study done by Liu and colleagues also showed that without the addition of osteogenic supplements, the best SMS value for optimizing proliferation was $12 \%$ for HPDLSCs [11]. In contrast, upon application of tension at the same level, HPDLSCs cultured in osteogenic media exhibited decrease in proliferation [8].

In conclusion, tension can regulate the differentiation and proliferation of PDLSCs in vitro. For PDLSCs from healthy donors, tension with a magnitude of $12 \%$ could increase osteogenic differentiation and proliferation of PDLSCs and tension above $12 \%$ would upregulate the function of PDLSCs to regulate osteoclast differentiation. Thus, the best SMS value for balance osteogenesis and osteoclastogenesis was $12 \%$, while the optimal force for PPDLSCs was $8 \%$. This could explain why lighter force should be used during OTM, especially for periodontitis patients. In addition, similar force magnitudes could lead to different differentiation paths, which may be due to the different culture medium and mechanical devices [9-11].

4.2. Compression. Static compression is commonly used in vitro to mimic the force on the compressive side during OTM. It has been reported to result in the altered morphology and the differentiation of PDLSCs.

It was found that the morphology and osteogenic gene expression of hPDLSCs responded to compression and would recover after force withdrawal [6]. Upon application of compression at $1 \mathrm{~g} / \mathrm{cm}^{2}$ for $12 \mathrm{~h}$ and $24 \mathrm{~h}$, hPDLSCs obtained significantly denser actin distribution and elongated morphology. In addition, the expression of collagen matrix and osteogenic marker (Col-I) in hPDLSCs was suppressed, resulting in a broken and disorganized pattern of PDL collagen. However, both the morphology and decreased gene expression recovered after force withdrawal.
To simulate the compression to hPDLSCs during the OTM process, Zhang and colleagues used a hydrauliccontrolled cellular strain element [5]. The compression on cells was produced by continuous compression of $2 \% \mathrm{CO}_{2}$ and $95 \% \mathrm{~N}_{2}$. Exposed to $100 \mathrm{kPa}$ static hydraulic pressures, hPDLSCs exhibited increased osteogenic differentiation after applying force for $1 \mathrm{~h}$, while osteogenic differentiation of hPDLSCs remained or reduced after $12 \mathrm{~h}$. On the contrary, the ratio of RANKL/OPG was decreased after $1 \mathrm{~h}$, while upregulated after $12 \mathrm{~h}$, which meant that the oclastogenesis was inhibited after $1 \mathrm{~h}$ but promoted after $12 \mathrm{~h}$.

In general, short-term compression (applying force for $1 \mathrm{~h}$ ) could promote osteogenic differentiation of PDLSCs, while long-term compression (applying force for $12 \mathrm{~h}$ or longer) inhibits osteogenesis and promotes osteoclastogenesis by increasing the RANKL/OPG ratio. These may be one of the reasons why compression causes bone resorption and acts as the rate-limiting step.

4.3. Vibration. Applying low-magnitude, high-frequency (LMHF) vibration at $0.3 \mathrm{~g}$ with a frequency of $10-180 \mathrm{~Hz}$ for 30 mins to hPDLSCs, Zhang and colleagues confirmed that there was a tendency to reduce the proliferation and upregulate the osteogenic differentiation of hPDLSCs as the frequency of stimulation increased, which peaked at $50 \mathrm{~Hz}$ [12]. In another study, they processed the LMHF vibration at $50 \mathrm{~Hz}$ with a magnitude of $0.05-0.9 \mathrm{~g}$ for hPDLSCs and found that vibration was most beneficial for the osteogenesis of hPDLSCs at $50 \mathrm{~Hz}$ with $0.3 \mathrm{~g}$ magnitude [13]. In conclusion, LMHF vibration decreases the proliferation and promotes the osteogenesis of hPDLSCs in frequency-dependent and magnitude-dependent manners and the optimal frequency and magnitude are $50 \mathrm{~Hz}$ and $0.3 g$, respectively $[12,13]$.

4.4. Others. With a frequency in the low-megahertz range $(1-3 \mathrm{MHz})$, low-intensity pulsed ultrasound (LIPUS) is widely used as a safe and minimally noninvasive application for regeneration and tissue repair [28]. Indeed, a study showed that the application of LIPUS accelerated the healing of periodontal tissue in vivo [29]. In another study, treated with $1 \mathrm{MHz}$ LIPUS for 5/20 minutes, rPDLSCs exhibited increased proliferation, indicating that LIPUS can promote the expansion of PDLSCs [14].

Three-dimensional (3D) dynamic simulation of microgravity induced by a rotary system also had effects on hPDLSCs, and it would benefit their proliferation and osteogenic differentiation [15]. Simultaneously, the morphology of hPDLSCs was changed from a triangular or spindle shape to a sphere shape body.

\section{Molecules Linking Applied Force with the Fate of PDLSCs In Vitro}

As mentioned above, applied force is a key regulator for the fate of PDLSCs. Understanding the cellular signaling pathway involved in the mechanical response of PDLSCs is essential for the future improvements in orthodontic therapy. 


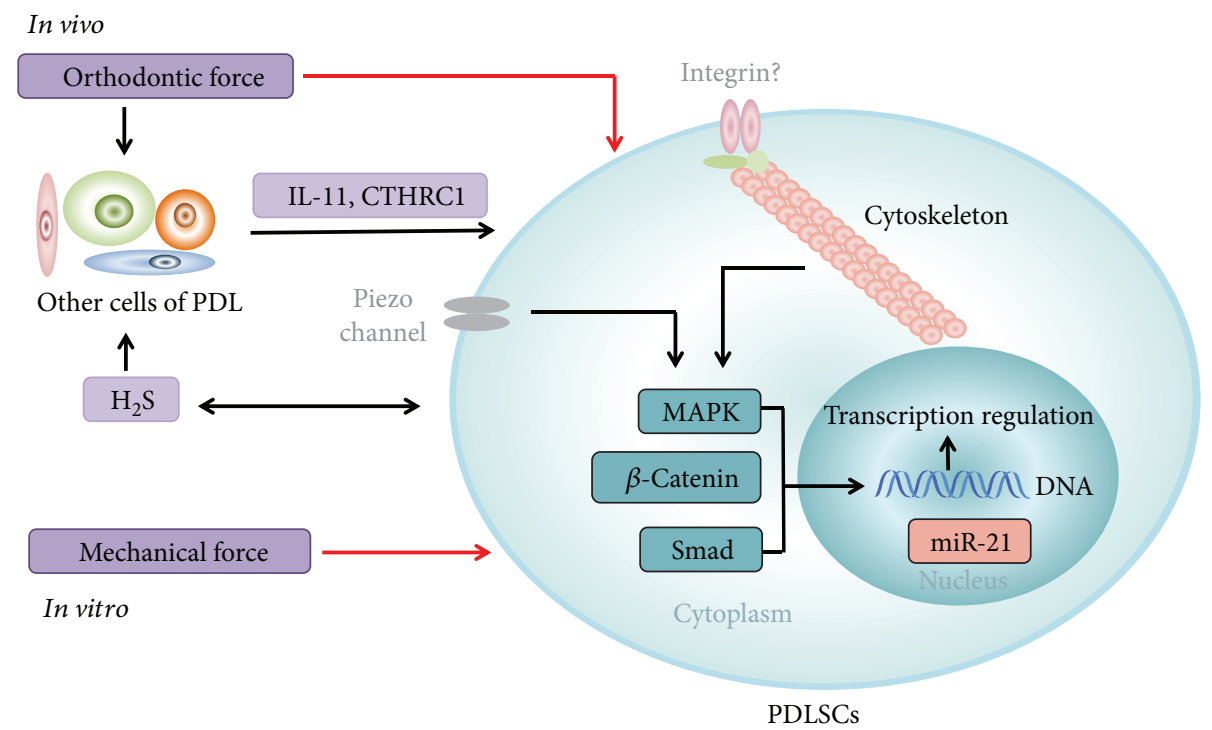

FIgURE 1: The mechanical forces could act directly on PDLSCs both in vitro and in vivo (red arrows), while in vivo, they may influence PDLSCs indirectly through the factors secreted by other PDLCs (black arrows). A variety of molecules is involved in regulating the fate of PDLSCs after the application of force. IL-11, CTHRC1, miR-21, and $\mathrm{H}_{2} \mathrm{~S}$ have been shown to help in transducing orthodontic force signals to PDLSCs. The cytoskeleton and MAPK, TGF- $\beta / \mathrm{Smad}$, and $\mathrm{Wnt} / \beta$-catenin pathways also play important roles in it.

5.1. Mechanosensors. It is not yet clear how cells recognize the mechanical force and convert it into cellular signals. Various mechanosensors have been proposed, including the cytoskeleton, membrane channels, primary cilia, focal adhesions, and gap junctions [30]. However, just the cytoskeleton and Piezo channel have been studied in PDLSCs.

The cytoskeleton consists mainly of actin, microtubules, and intermediate filaments. As the continuous structure between cell membrane and chromosome, the cytoskeleton supplies a structural framework for cells. Mechanical force may change cytoskeletal tension by the change of cell shape [31]. After LMHF mechanical stimulation, the F-actin fibers in hPDLSCs became clearer and thicker [13]. Furthermore, the level of cytoskeletal remodeling influenced the mechanically driven osteogenic commitment of PDLSCs, which was correlated to the magnitude of the applied vibration stimulus, suggesting that the cytoskeleton was involved in transmitting mechanical forces into cells [13].

Piezo is a mechanosensitive membrane ion channel. Mechanical stress can regulate the differentiation of stem cell in the midgut of mature Drosophila via the tension-activated Piezo channel [32]. Gao and colleagues also showed that the Piezo channel was likely to play an important role in conducting ultrasound-related signals in dental pulp stem cells [33]. In the same study, however, they observed that the Piezo channel was not associated with LIPUS-stimulated rPDLSC proliferation.

5.2. Mechanotransduction Pathways. Multiple pathways mediate the response of PDLSCs to mechanical stimulation. The functions of the mitogen-activated protein kinase (MAPK), transforming growth factor- $\beta$ (TGF- $\beta$ ), and Wnt/ $\beta$-catenin pathways are discussed in more detail below.

Force-induced proliferation of rPDLSCs can involve the activation of MAPK signaling pathways. When LIPUS was applied to rPDLSCs, the c-Jun N-terminal kinases (JNK) MAPK signaling was immediately activated and p38 MAPK kinase was activated 4 hours after the exposure [14]. At the same time, the inhibitions of JNK and p38 reduced LIPUSassociated proliferation of rPDLSCs.

TGF- $\beta$ is a critical molecule in extracellular matrix remodeling and tissue homeostasis [34]. When hPDLSCs are exposed to compression, the expressions of TGF- $\beta 1$ and TGF- $\beta 3$ in hPDLSCs were suppressed after force treatment and recovered following force withdrawal, which was consistent with the alteration of Col-I expression and PDL collagen [6]. Besides, blocking the TGF- $\beta /$ Smad pathway could inhibit the recovery of Col-I expression and PDL collagen during early orthodontic relapse [6].

The Wnt/ $\beta$-catenin pathway plays important roles in the regulation of osteogenic differentiation of PDLSCs. The canonical pathway is involved in translocating $\beta$-catenin into the nucleus. Zhang and colleagues found that compression activated the Wnt/ $\beta$-catenin pathway and increased the levels of glycogen synthase kinase $3 \beta$ (GSK-3 $\beta$ ), active- $\beta$ catenin proteins, and phospho-GSK-3 $\beta$ in hPDLSCs [5]. Furthermore, Dickkopf-related protein 1, the inhibitor of the canonical Wnt pathway, could block the osteogenic differentiation and reinstate the ratio of RANKL/OPG following force treatment.

\section{Conclusion and Perspective}

PDLSCs play an important role in OTM. rPDLSCs may be reactivated on both sides during orthodontic force treatment and participate in the orthodontic relapse process. In vitro, both tension and compression can regulate osteogenic differentiation and proliferation of PDLSCs, which is consistent with in vivo experiments. Under the optimal magnitude, which is $12 \%$ for HPDLSCs and $8 \%$ for PPDLSCs, tension 
could increase osteogenic differentiation and proliferation of PDLSCs. And it would upregulate the function of PDLSCs to regulate osteoclast differentiation when the magnitude is above optimal. Short-term compression could promote osteogenic differentiation of PDLSCs, while long-term compression inhibits osteogenesis and promotes osteoclastogenesis. Besides, LMHF vibration decreases the proliferation and promotes the osteogenesis of hPDLSCs and the optimal frequency and magnitude are $50 \mathrm{~Hz}$ and $0.3 \mathrm{~g}$, respectively. A variety of mechanosensors and pathways are involved in it, including cytoskeleton, MAPK signaling, TGF- $\beta /$ Smad, and Wnt $/ \beta$-catenin pathways. IL-11, CTHRC1, MiR-21, and $\mathrm{H}_{2} \mathrm{~S}$ have also been shown to be important to transduce orthodontic force signals into PDLSCs in vivo (Figure 1).

This article reviewing the relationship between PDLSCs and mechanical force is crucial to understand the healing process during orthodontic tooth movement, which may help orthodontics to control the orthodontic procedure more effectively. As mentioned above, excessive force will damage the function of PDLSCs, so lighter force should be used during OTM, especially for periodontitis patients. Due to their proliferation and differentiation potential, PDLSCs could potentially be used for the reconstruction of periodontal tissues, which might accelerate the procedure of orthodontic treatment.

However, there are several important issues that still need to be investigated. Firstly, it needs to seed PDLSCs on a culture plate of proper rigidity or three-dimension scaffolds in vitro to find the optimal method to mimic the nature environment during OTM. In addition, investigating the role of extracellular matrix and other potential molecules such as integrin and Erk1/2 MAPK, which have been found to be mechanotransduction in PDLCs [35], will help in figuring out how the force is transduced into PDLSCs. Furthermore, whether the effects of PDLSCs on peripheral blood mononuclear cell and $\mathrm{T}$ cell are regulated by orthodontic force and why the PPDLSCs respond differently to force need to be addressed. Finally, investigating the function of PDLSCs in orthodontic root resorption and orthodontic relapse will contribute to understanding OTM and control the major clinical challenges in orthodontic treatment.

\section{Conflicts of Interest}

The authors of this review clarify no conflict of interest.

\section{Acknowledgments}

This work was supported by the Beijing Municipal Natural Science Foundation (7182182 to Ruili Yang), the National Natural Science Foundation of China (no. 81470717 to Yan-heng Zhou and no. 81600865 to Ruili Yang), and the Young Elite Scientist Sponsorship (YESS) Program by CAST Innovation Foundation (2017QNRC001).

\section{References}

[1] V. Krishnan and Z.'e. Davidovitch, "Cellular, molecular, and tissue-level reactions to orthodontic force," American Journal of Orthodontics and Dentofacial Orthopedics, vol. 129, no. 4, pp. 469.e1-469.e32, 2006.

[2] G. E. Wise and G. J. King, "Mechanisms of tooth eruption and orthodontic tooth movement," Journal of Dental Research, vol. 87, no. 5, pp. 414-434, 2008.

[3] B.-M. Seo, M. Miura, S. Gronthos et al., "Investigation of multipotent postnatal stem cells from human periodontal ligament," The Lancet, vol. 364, no. 9429, pp. 149155, 2004.

[4] N. Wada, D. Menicanin, S. Shi, P. M. Bartold, and S. Gronthos, "Immunomodulatory properties of human periodontal ligament stem cells," Journal of Cellular Physiology, vol. 219, no. 3, pp. 667-676, 2009.

[5] L. Zhang, W. Liu, J. Zhao et al., "Mechanical stress regulates osteogenic differentiation and RANKL/OPG ratio in periodontal ligament stem cells by the Wnt/ $\beta$-catenin pathway," Biochimica et Biophysica Acta (BBA) - General Subjects, vol. 1860, no. 10, pp. 2211-2219, 2016.

[6] L. Feng, R. Yang, D. Liu et al., "PDL progenitor-mediated PDL recovery contributes to orthodontic relapse," Journal of Dental Research, vol. 95, no. 9, pp. 1049-1056, 2016.

[7] N. Tang, Z. Zhao, L. Zhang et al., "Up-regulated osteogenic transcription factors during early response of human periodontal ligament stem cells to cyclic tensile strain," Archives of Medical Science, vol. 8, no. 3, pp. 422-430, 2012.

[8] T. Shen, L. Qiu, H. Chang et al., "Cyclic tension promotes osteogenic differentiation in human periodontal ligament stem cells," International Journal of Clinical and Experimental Pathology, vol. 7, no. 11, pp. 7872-7880, 2014.

[9] D. Pelaez, Z. Acosta Torres, T. K. Ng, K. W. Choy, C. P. Pang, and H. S. Cheung, "Cardiomyogenesis of periodontal ligament-derived stem cells by dynamic tensile strain," Cell and Tissue Research, vol. 367, no. 2, pp. 229-241, 2017.

[10] J. Chen, W. Zhang, L. J. Backman, P. Kelk, and P. Danielson, "Mechanical stress potentiates the differentiation of periodontal ligament stem cells into keratocytes," British Journal of Ophthalmology, vol. 102, no. 4, pp. 562-569, 2018.

[11] J. Liu, Q. Li, S. Liu et al., "Periodontal ligament stem cells in the periodontitis microenvironment are sensitive to static mechanical strain," Stem Cells International, vol. 2017, Article ID 1380851, 13 pages, 2017.

[12] C. Zhang, J. Li, L. Zhang et al., "Effects of mechanical vibration on proliferation and osteogenic differentiation of human periodontal ligament stem cells," Archives of Oral Biology, vol. 57, no. 10, pp. 1395-1407, 2012.

[13] C. Zhang, Y. Lu, L. Zhang et al., "Influence of different intensities of vibration on proliferation and differentiation of human periodontal ligament stem cells," Archives of Medical Science, vol. 3, no. 3, pp. 638-646, 2015.

[14] Q. Gao, A. D. Walmsley, P. R. Cooper, and B. A. Scheven, "Ultrasound stimulation of different dental stem cell populations: role of mitogen-activated protein kinase signaling," Journal of Endodontia, vol. 42, no. 3, pp. 425-431, 2016.

[15] S. Li, Z. Ma, Z. Niu et al., "NASA-approved rotary bioreactor enhances proliferation and osteogenesis of human periodontal ligament stem cells," Stem Cells and Development, vol. 18, no. 9, pp. 1273-1282, 2009.

[16] T. J. Franzen, P. Brudvik, and V. Vandevska-Radunovic, "Periodontal tissue reaction during orthodontic relapse in rat molars," European Journal of Orthodontics, vol. 35, no. 2, pp. 152-159, 2013. 
[17] X. Du and D. A. Williams, "Interleukin-11: review of molecular, cell biology, and clinical use," Blood, vol. 89, no. 11, pp. 3897-3908, 1997.

[18] H. Matsumura, Y. Nakayama, H. Takai, and Y. Ogata, "Effects of interleukin-11 on the expression of human bone sialoprotein gene," Journal of Bone and Mineral Metabolism, vol. 33, no. 2, pp. 142-153, 2015.

[19] S. Monnouchi, H. Maeda, A. Yuda et al., "Mechanical induction of interleukin-11 regulates osteoblastic/cementoblastic differentiation of human periodontal ligament stem/ progenitor cells," Journal of Periodontal Research, vol. 50, no. 2, pp. 231-239, 2015.

[20] S. Takeshita, T. Fumoto, K. Matsuoka et al., "Osteoclastsecreted CTHRC1 in the coupling of bone resorption to formation," The Journal of Clinical Investigation, vol. 123, no. 9, pp. 3914-3924, 2013.

[21] C. Wang, W. Gu, B. Sun et al., "CTHRC1 promotes osteogenic differentiation of periodontal ligament stem cells by regulating TAZ," Journal of Molecular Histology, vol. 48, no. 4, pp. 311319, 2017.

[22] F. L. Wei, J. H. Wang, G. Ding et al., "Mechanical forceinduced specific MicroRNA expression in human periodontal ligament stem cells," Cells Tissues Organs, vol. 199, no. 5-6, pp. 353-363, 2014.

[23] F. Wei, D. Liu, C. Feng et al., "microRNA-21 mediates stretch-induced osteogenic differentiation in human periodontal ligament stem cells," Stem Cells and Development, vol. 24, no. 3, pp. 312-319, 2015.

[24] N. Chen, B. D. Sui, C. H. Hu et al., "microRNA-21 contributes to orthodontic tooth movement," Journal of Dental Research, vol. 95, no. 12, pp. 1425-1433, 2016.

[25] R. Yang, Y. Liu, and S. Shi, "Hydrogen sulfide regulates homeostasis of mesenchymal stem cells and regulatory T cells," Journal of Dental Research, vol. 95, no. 13, pp. 1445-1451, 2016.

[26] F. Liu, F. Wen, D. He et al., "Force-induced $\mathrm{H}_{2} \mathrm{~S}$ by PDLSCs modifies osteoclastic activity during tooth movement," Journal of Dental Research, vol. 96, no. 6, pp. 694-702, 2017.

[27] S. G. Kim, T. Akaike, T. Sasagaw, Y. Atomi, and H. Kurosawa, "Gene expression of type I and type III collagen by mechanical stretch in anterior cruciate ligament cells," Cell Structure and Function, vol. 27, no. 3, pp. 139-144, 2002.

[28] F. Padilla, R. Puts, L. Vico, and K. Raum, "Stimulation of bone repair with ultrasound: a review of the possible mechanic effects," Ultrasonics, vol. 54, no. 5, pp. 1125-1145, 2014.

[29] H. Ikai, T. Tamura, T. Watanabe et al., "Low-intensity pulsed ultrasound accelerates periodontal wound healing after flap surgery," Journal of Periodontal Research, vol. 43, no. 2, pp. 212-216, 2008.

[30] J. C. Chen and C. R. Jacobs, "Mechanically induced osteogenic lineage commitment of stem cells," Stem Cell Research \& Ther$a p y$, vol. 4, no. 5, p. 107, 2013.

[31] R. McBeath, D. M. Pirone, C. M. Nelson, K. Bhadriraju, and C. S. Chen, "Cell shape, cytoskeletal tension, and RhoA regulate stem cell lineage commitment," Developmental Cell, vol. 6, no. 4, pp. 483-495, 2004.

[32] L. He, G. Si, J. Huang, A. D. T. Samuel, and N. Perrimon, "Mechanical regulation of stem-cell differentiation by the stretch-activated Piezo channel," Nature, vol. 555, no. 7694, pp. 103-106, 2018.

[33] Q. Gao, P. R. Cooper, A. D. Walmsley, and B. A. Scheven, "Role of Piezo channels in ultrasound-stimulated dental stem cells," Journal of Endodontia, vol. 43, no. 7, pp. 1130-1136, 2017.

[34] T. Furumatsu, E. Matsumoto, T. Kanazawa et al., "Tensile strain increases expression of CCN2 and COL2A1 by activating TGF- $\beta$-Smad $2 / 3$ pathway in chondrocytic cells," Journal of Biomechanics, vol. 46, no. 9, pp. 1508-1515, 2013.

[35] N. Ziegler, A. Alonso, T. Steinberg et al., "Mechano-transduction in periodontal ligament cells identifies activated states of MAP-kinases p42/44 and p38-stress kinase as a mechanism for MMP-13 expression," BMC Cell Biology, vol. 11, no. 1, p. 10,2010 . 


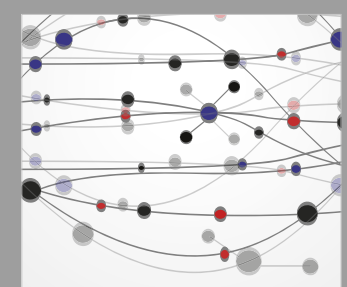

The Scientific World Journal
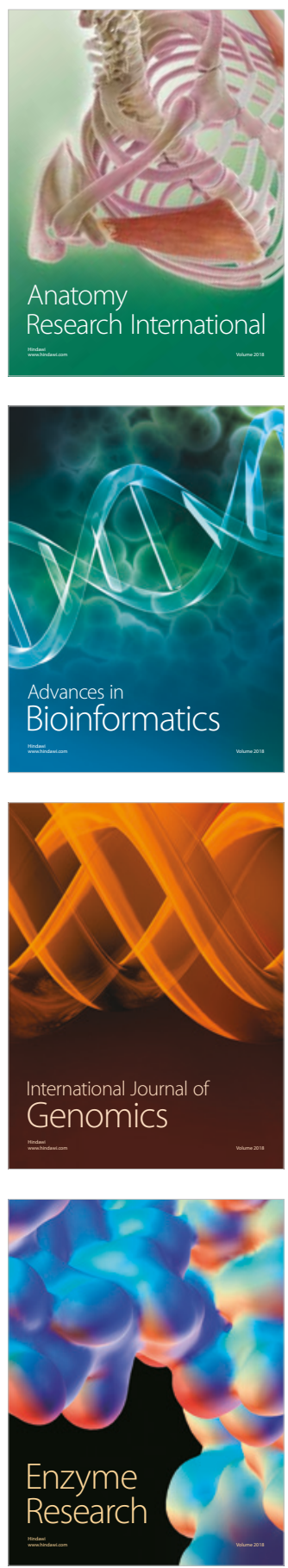
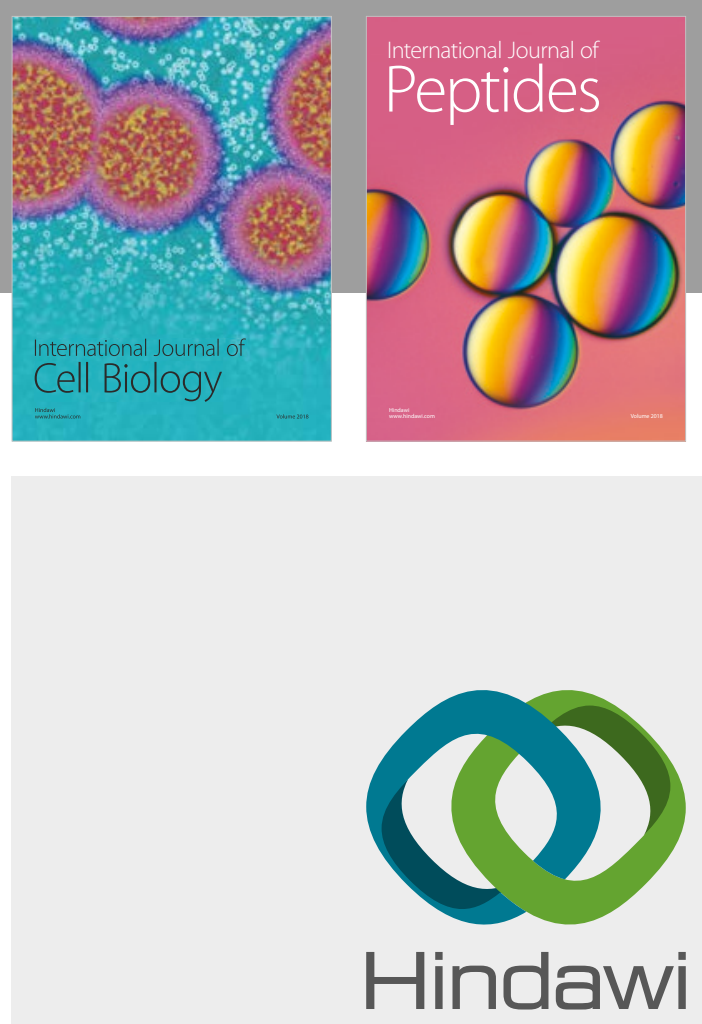

Submit your manuscripts at

www.hindawi.com
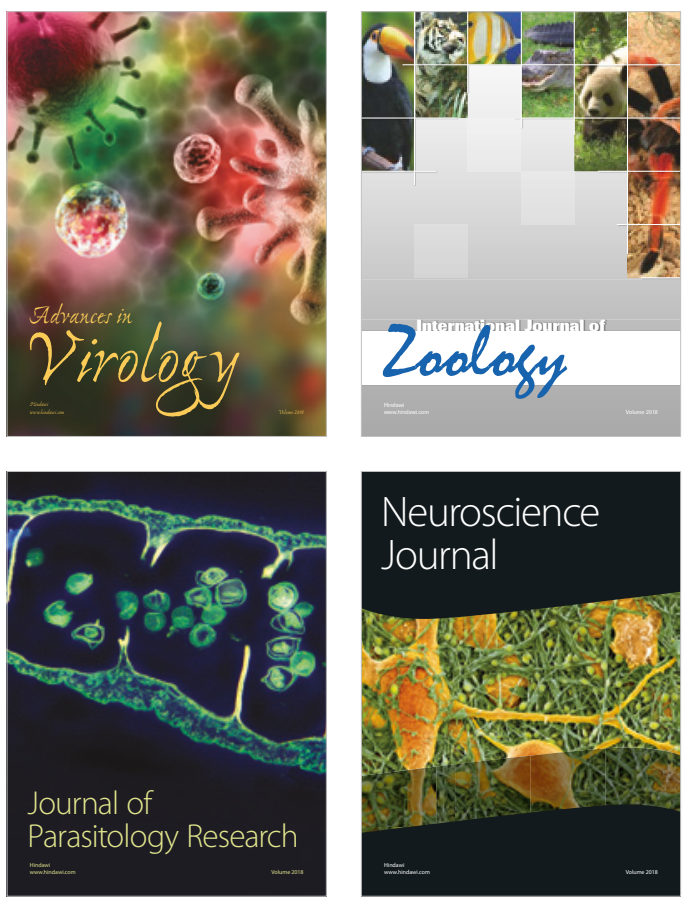
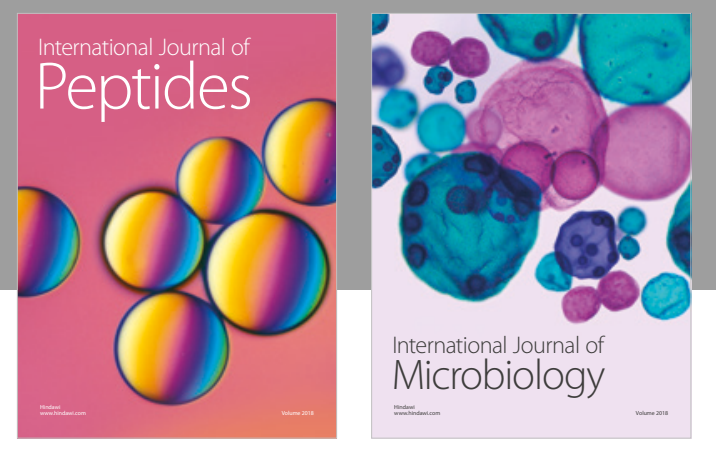

nternational Journal of Microbiology
Journal of
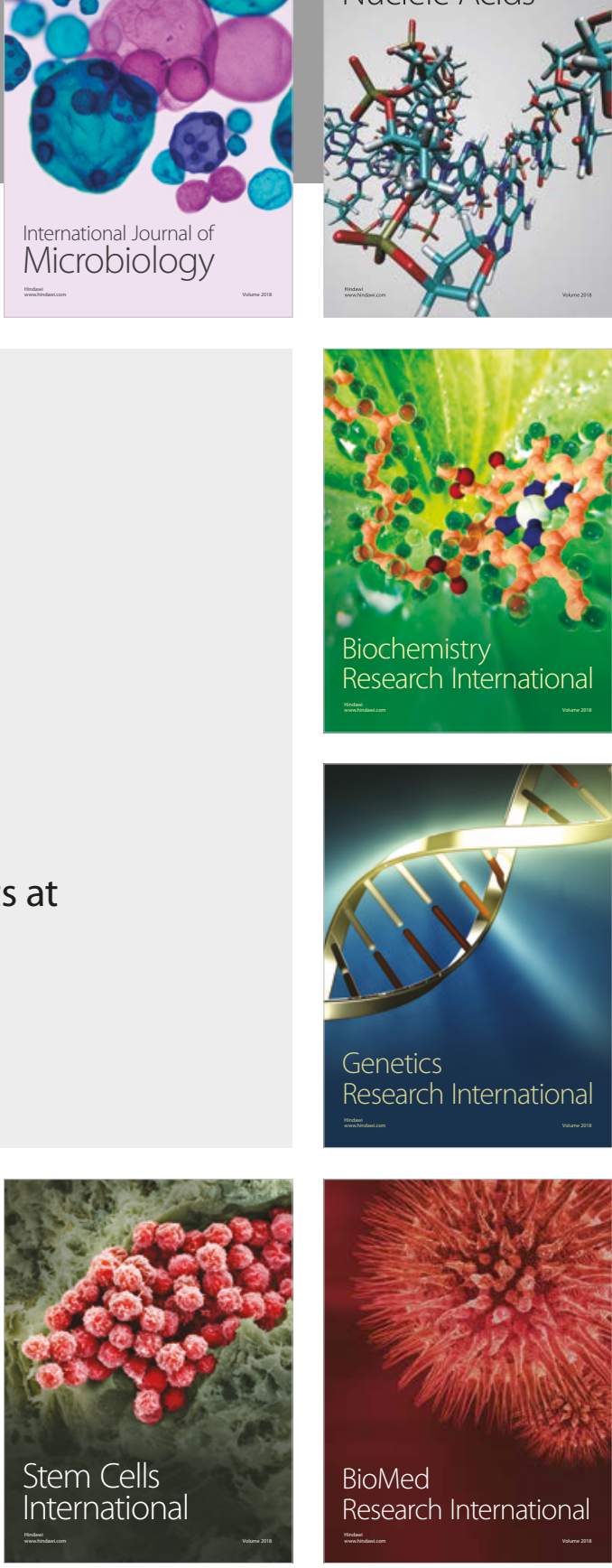
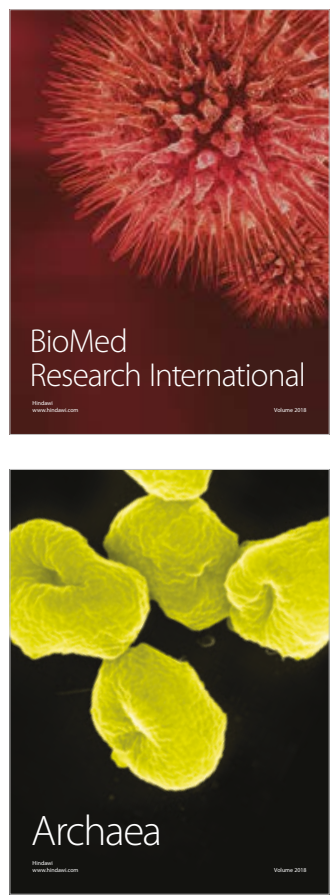